\title{
Achieved Competencies and Satisfaction in Temporomandibular Disorders and Orofacial Pain Education
}

\author{
Sara Nordin, DDS \\ Clinical Assistant \\ Department of Orofacial Pain and Jaw \\ Function \\ Faculty of Odontology \\ Malmö University \\ Malmö, Sweden
}

\author{
Andreas Dawson, DDS, Odont Dr, \\ $\mathrm{PhD}$ \\ Consultant in Orofacial pain \\ Centre for Oral Rehabilitation \\ Östergötland County Council \\ Linköping, Sweden \\ Postgraduate Student \\ Department of Orofacial Pain and Jaw \\ Function \\ Faculty of Odontology \\ Malmö University \\ Malmö, Sweden
}

\author{
Ewa Carin Ekberg, DDS, Odont Dr, \\ PhD \\ Professor \\ Department of Orofacial Pain and Jaw \\ Function \\ Faculty of Odontoloy \\ Malmö University \\ Malmö, Sweden
}

\section{Correspondence to:}

Dr Andreas Dawson

Centre for Oral Rehabilitation

Östergötland County Council

Torkelbergsgatan 11

58185 Linköping

Sweden

Email: andreas.dawson@

regionostergotland.se

C2016 by Quintessence Publishing Co Inc.
Aims: To assess dental students' achieved competencies and perceived satisfaction with their temporomandibular disorders (TMD) and orofacial pain education and to compare these with the results of their final examination in TMD and orofacial pain. Methods: Dental students from two consecutive classes (2011/2012 and 2012/2013) at the Department of Orofacial Pain and Jaw Function at the dental school in Malmö, Sweden completed two self-evaluations, one at the beginning of semester seven and one at the end of semester eight. The questionnaire that they were given concerned achieved competencies and satisfaction with education in TMD and orofacial pain. Items focused on anatomy, physiology, and clinical training. Students estimated their competence and satisfaction on a numeric rating scale and described their idea of treating TMD and orofacial pain patients on a verbal rating scale. Outcome variables were tested with paired samples $t$ test for differences over time and independent samples $t$ test for between-class comparisons; both were adjusted for multiple testing with Bonferroni correction. Results: Significant improvement in all items was observed for achieved competencies and satisfaction in both classes between semester seven and semester eight $(P<.05)$. No differences in competencies or satisfaction occurred between classes at the end of the clinical course in semester eight $(P>05)$. Conclusion: This study has shown that expansion in undergraduate TMD and orofacial pain education at the dental school in Malmö has allowed all students to develop the same level of competence, independent of prior experience. The study also pointed out that continuous evaluation and enhancement of TMD and orofacial pain education in undergraduate dental education is beneficial. $J$ Oral Facial Pain Headache 2016;30:156-164. doi: 10.11607/ofph.1471

Keywords: education, problem-based learning, professional competence, temporomandibular joint disorders

n 2009, Watt-Watson et al discussed the discrepancy in pain education between different health care-related programs in Canada. ${ }^{1}$ They concluded that the number of hours dedicated to pain education differed widely between the programs. At dental faculties, the mean time assigned to pain education was 15 hours, and at veterinary schools, 87 hours.

In 2011, Sessle noted gaps in knowledge on chronic pain as well as variations in how newly gained knowledge about pain and standards of practice are implemented, and that chronic pain conditions are present in epidemic proportions in most countries and are associated with significant socioeconomic consequences. ${ }^{2} \mathrm{He}$ concluded that the understanding of pain and its management needs much improvement and the most crucial needs are to raise pain awareness, enhance pain education, improve access to pain care, and increase funding for pain research. ${ }^{2}$

The Faculty of Odontology at Malmö University uses a problem-based learning (PBL) pedagogic model. The 5-year undergraduate dental education, comprising 10 semesters altogether, is based on oral conditions prevalent in Swedish society, according to the Malmö model. ${ }^{3}$ These conditions are put into context in a way that integrates learning with clinical practice: based on the model of a spiral curriculum, the 
complexity of the learning context, theoretic knowledge, and clinical competence gradually increases. Theoretic knowledge is acquired primarily through working with clinically related cases in a study group, in which a small group of students and a tutor participate. During the first 3 years (the first six semesters) study groups meet twice a week, and thereafter, once a week. Starting in semester eight, tutors do not attend the meetings. The theoretic knowledge is intended to prepare students for clinic duty, where they can build on their previous knowledge in a clinical context.

Temporomandibular disorders (TMD) and orofacial pain are two common problems in public health. The prevalence of TMD among adults is about 10\% and pain is the most common reason to seek treatment for TMD. ${ }^{4}$ Since 1 out of 10 patients suffers from TMD and orofacial pain, dental students need to have good knowledge in these areas. Vallon and Nilner investigated the perception of achieved competencies in TMD and orofacial pain among undergraduates and graduates of the dental program. It was observed that most competencies in TMD and orofacial pain increased significantly during training..$^{5,6}$

The focus on pain education in undergraduate dental education at Malmö University has grown in recent years. Students begin learning about the physiologic mechanisms and biologic effects of pain in the second semester. Pain education continues during the fourth, seventh, eighth, ninth, and tenth semesters. Acute oral pain conditions are discussed during the first 3 years, while TMD and orofacial pain is introduced in semester seven and learned in-depth in semester nine. Guided by the conclusions of WattWatson et al ${ }^{1}$ and Sessle, ${ }^{2}$ the TMD and orofacial pain education in semesters seven and eight has been revised and further enhanced. ${ }^{1,2}$ After the TMD and orofacial pain education, the students should be able to identify, examine, diagnose, treat, and evaluate TMD and orofacial pain patients, and also identify patients who are in need of specialist care.

The aim of this study was to assess dental students' achieved competencies and perceived satisfaction with their TMD and orofacial pain education, and to compare these with the results of their final examination in TMD and orofacial pain. The following hypotheses were tested: (1) the students' achieved competencies and satisfaction with TMD and orofacial pain education will increase over the seventh and eight semesters; (2) no differences in competencies and satisfaction will occur between classes; and (3) the outcome of the course examination in semester seven will correlate with students' subjective experiences of their achieved competencies in TMD and orofacial pain and their satisfaction with the education.

\section{Materials and Methods}

\section{Study Design}

In a prospective longitudinal study, two classes of dental students (the class of 2011/2012 and the class of $2012 / 2013$ ) were each invited to respond to a self-evaluation on two occasions during their education at Malmö University. Mean age \pm standard deviation (SD) in each class was $24.5 \pm 2.4$ years (class of 2011/2012) and $25.3 \pm 4.0$ years (class of 2012/2013). The first evaluation occurred at the start of semester seven and the second at the end of the clinical course in semester eight. Students were informed about the project and its purpose and were invited to participate on a voluntary and anonymous basis. Therefore, this study was exempted from ethical approval. This study follows the World Medical Association Declaration of Helsinki guidelines.

\section{Theoretic Knowledge and Preclinical Course}

In the seventh semester, undergraduate dental students gain theoretic knowledge about TMD pain and headache, analgesic mechanisms, and treatment options for TMD pain by participating in a study group (Figs 1a and 1b). In tandem with the study group, seventh-semester students participate in a preclinical course in TMD and orofacial pain to prepare them for student clinic duty at the end of the semester. This preclinical course teaches diagnostic methods and treatment options for TMD and orofacial pain (Table 1, Fig 2).

In semester eight, the theoretic knowledge acquired in semester seven is applied to clinical cases of children and adolescents with TMD and orofacial pain.

\section{Clinical Practice in the Student Clinic}

Patients in the student clinic are those who have been referred from general dentists or physicians. Students begin with patient care after an oral examination at the end of semester seven to ensure their preparedness for clinic duty during semester eight (Table 1, Fig 2).

After the eighth semester, the dental students are no longer scheduled at the Department of Orofacial Pain and Jaw Function's student clinic. They must then screen their patients for TMD and orofacial pain during duty at other departments' student clinics. In the tenth semester, students are assigned to public dental clinics once a week to practice general dentistry. It is essential for them to be able to identify patients with TMD and other types of orofacial pain in general practice. Therefore, the students are taught to use three screening questions for TMD and orofacial pain when taking an oral history. Nilsson et al established these questions, and two of the three questions are validated 


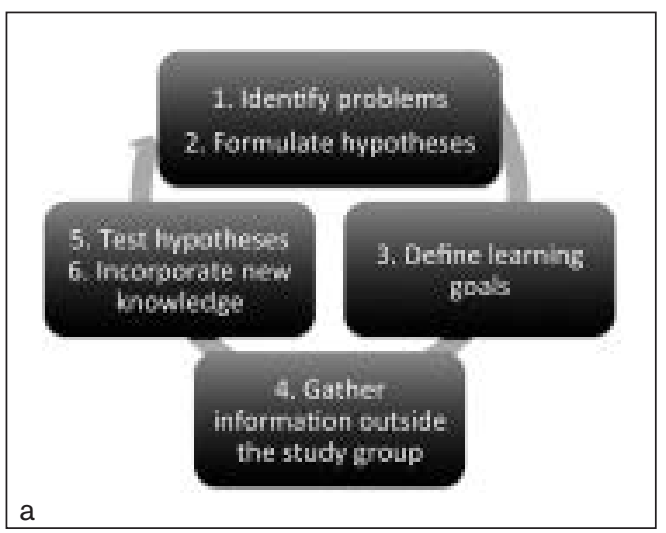

45-year-old Darko is a taxi driver who in the past year has been troubled by a feeling of heaviness in the head and pain in front of the ears several times a week. Darko's headache and ear pain are alleviated most of the time with the help of aspirin, but it gives him stomach pain. Now Darko's ear pain has become acute, and in the past few days, he has had severe pain day and night. Darko gets an emergency appointment with his family doctor, who examines his ears without finding any explanation of his ear pain and, therefore, asks Darko to contact his dentist. Darko has now got an appointment with you and Darko wants an explanation for his pain and a prescription for pills that can provide pain relief.

Fig 1a Schematic of the problem-based learning process when solving a clinically related case in the study group. Students construct problems and hypotheses appropriate for the case, and after acquisition of knowledge they try to solve the problems by confirming or denying their hypotheses. Modified after Rohlin et al. ${ }^{3}$

Fig 1b Clinical case from study group in semester 7: Darko. Example of one of the clinical cases presented to the study group in semester seven.

\section{Table 1 Theoretic Knowledge and Preclinical Courses for TMD and Orofacial Pain Students in the Seventh Semester}

Study group

Seminars

Auscultation

$\mathrm{RDC} / \mathrm{TMD}$ and $\mathrm{DC} / \mathrm{TMD}$

Patient care

Clinical hour

Workshop
The purpose of study groups is to create a multidisciplinary approach to pain education and to teach the student about the complexity of pain etiology, diagnosis, and treatment. Cases in the study group seldom focus solely on TMD and orofacial pain.

In semester seven, specialists in TMD and orofacial pain hold clinically oriented seminars in conjunction with the clinical cases in the study groups on pain and pharmaceuticals, reduced mouth opening capacity, systemic disorders, orofacial pain, and jaw function. There is also a multidisciplinary seminar discussing prosthetic treatment on a patient suffering from TMD and orofacial pain.

After an interactive presentation on pain mechanisms, students work in pairs at different stations in the pain laboratory. One station, for example, involves injecting hypertonic saline into the masseter muscle and then a discussion of the pain experience. The purpose is to initiate thoughts and understanding of pain thresholds, pain tolerance, referred pain, widespread pain, and pain reflexes.

Students train their ability to take case histories and set correct TMD diagnoses through interactive role play. They practice giving correct information about TMD, instructing patients in exercises to prevent or alleviate $T M D$, and writing the case histories with each other.

The instructor performs the TMD examination and students apply their theoretic knowledge and clinical competencies by making the diagnoses and writing the patient's case history as a group assignment.

Students are introduced to diagnostic methodology and criteria for TMD diagnoses. In the autumn of 2012, $\mathrm{DC} / \mathrm{TMD}$ replaced $\mathrm{RDC} / \mathrm{TMD}$.

In the preclinical course, students are introduced to various types of TMD and orofacial pain treatment options and how to validate them according to the recommendations in the Swedish National Guidelines from the Swedish National Board of Health. ${ }^{11}$ It is crucial that the treatment plan should be individually adapted to the patient's needs.

The last hour of the clinical pass, the clinical hour, is dedicated to discussions on patient-related matters so that students can share their experiences.

Various clinical hours have themes, such as:

- The prefabricated Relax appliance ${ }^{12}$; students practice on each other.

- Demonstrations of transcutaneous electrical nerve stimulation (TENS) and acupuncture by specially trained dental nurses; students have the opportunity to try both.

- An interactive presentation on the pain school; students discuss behavioral treatments. The pain school is a standardized program led by specially trained dental nurses, has a cognitive approach, and focuses on awareness and pain management.

- A visit to the Department of Oral Radiology; various types of diagnostic methods that would be suitable for examination of the temporomandibular joint are discussed. Specialists in radiology discuss when and why to consider such examination and how to use the results. 


\section{Pain education}

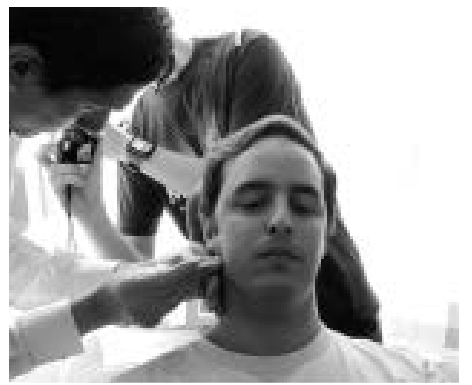

Pain laboratory

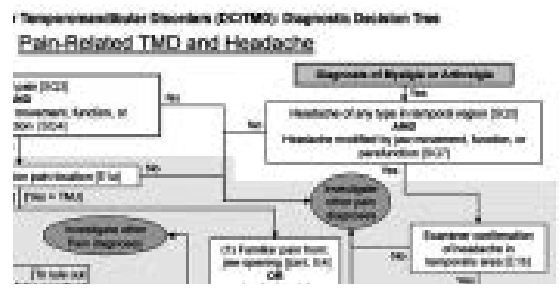

DC/TMD

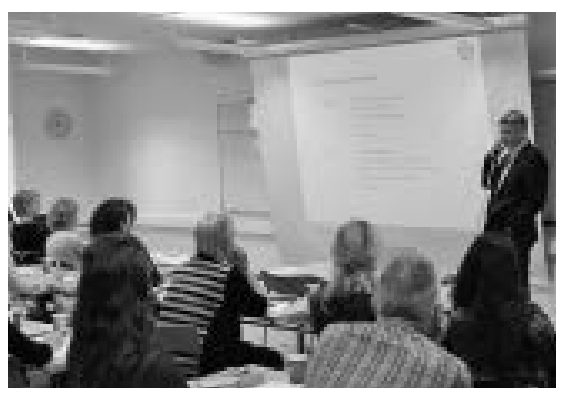

Seminars
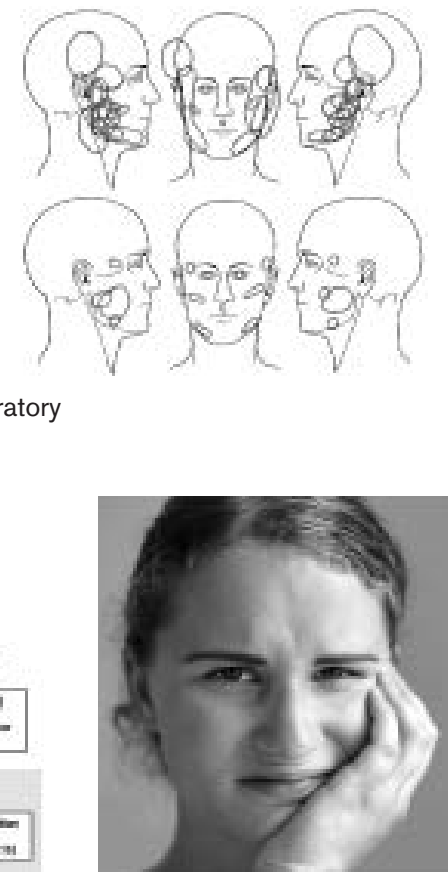

Patient care

Clinical hours

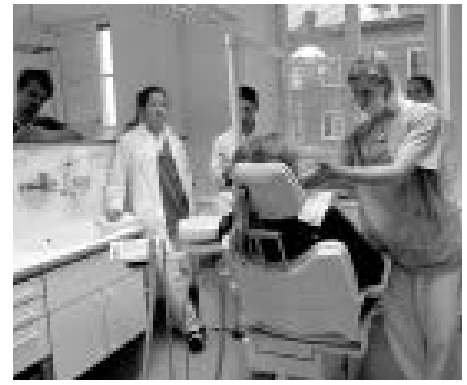

Auscultation

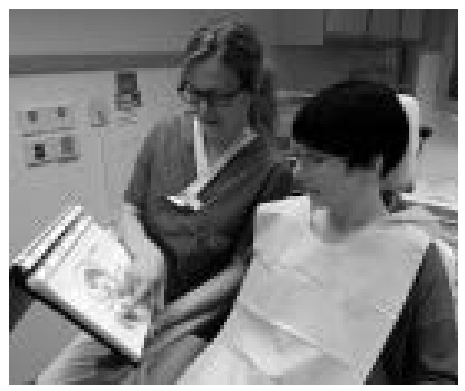

Role play

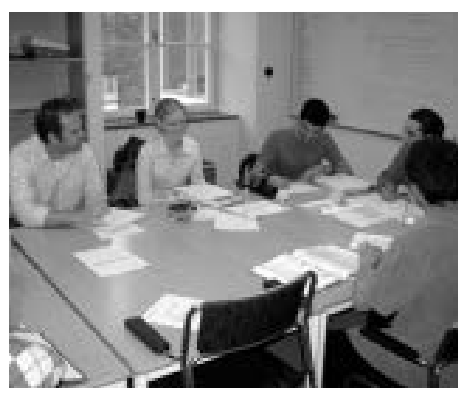

Study group

Fig 2 Pain education in undergraduate dental education, semesters seven and eight, Malmö University. The figure demonstrates the different aspects of pain education during the preclinical course in semester seven and during clinical training in semesters seven and eight at the Faculty of Odontology at Malmö University.

for children and adolescents. ${ }^{7}$ A positive response to at least one of the three questions indicates a need for further clinical examination for TMD and orofacial pain.

\section{Examination in TMD and Orofacial Pain}

The oral examination at the end of semester seven that is used to assess students' preparedness for clinic duty is multidisciplinary, and the topic of TMD and orofacial pain plays a significant part. The examination is a series of clinical cases for which students must solve problems and answer questions. The clinical cases are based on real patients seen at the departments, which most likely are generalizable for the patients that the students may encounter after graduation in general practice. Three calibrated teachers supervise, one each from the departments of Orofacial Pain and Jaw Function, Oral Prosthodontics, and Endodontics. After the examination, students describe how well the course objectives have been fulfilled in a mandatory course evaluation. Students evaluate course goals 
on a 5-point numeric rating scale (NRS) with the end-definitions "not fulfilled" and "fulfilled."

In semester eight, all students are required to make an oral case presentation in their clinical group. The prerequisite for taking the course examination is approval of the case presentation by a calibrated teacher. The course examination is similar to the one in semester seven but with much less emphasis on TMD and orofacial pain in adults; instead, the examination primarily concerns TMD and orofacial pain in children and adolescents. After each examination, the calibrated teachers have a consensus discussion and assess whether the students have passed or not using a dichotomous scale ("passed" versus "not passed").

\section{Research Diagnostic Criteria for TMD and Diagnostic Criteria for TMD}

When this study was initiated in 2011, specialists in TMD and orofacial pain and general dentists with a special interest in the subject supervised dental students at the student clinic. The Research Diagnostic Criteria for TMD (RDC/TMD) was the examination method of choice, and staff as well as students had been trained in its use. ${ }^{8}$ However, in late 2012, the updated version, Diagnostic Criteria for TMD (DC/TMD), was fully translated into Swedish by two of the authors (S.N., E.C.E.) and then implemented at the clinic after calibration. ${ }^{9}$ The reason for the change was that the DC/TMD is simpler and intended for the general dental practitioner. The cornerstones of the DC/TMD are familiar pain, referred pain, and headache attributed to TMD. The RDC/TMD and the DC/TMD both use Axis I (clinical TMD diagnoses) and Axis II (behavioral and psychosocial factors). In the DC/TMD, the clinician uses verbatim commands so that the examination will be reproducible. The diagnostic criteria in the DC/ TMD are validated and have moderate to high sensitivity and specificity for subdiagnoses of TMD. ${ }^{9}$

\section{Competencies and Satisfaction with the Education}

The dental students used a self-evaluation process to assess their achieved competencies and satisfaction with their education in TMD and orofacial pain, anatomy and physiology, and clinical training. Items in anatomy and physiology concerned the temporomandibular joint (TMJ), the jaw muscles, and pain. Items in clinical training concerned screening for TMD and orofacial pain, history and examination, diagnosis, prognosis, decision-making, and evaluation of treatment outcomes. A 0 to 10 NRS was used to assess achieved competencies and satisfaction (anchor definitions: "none"/"not satisfied" and "very high"/"very satisfied").

In addition, self-reported attitudes toward treating TMD and orofacial pain patients were assessed on a verbal rating scale (VRS) with the following adjec- tives: "interesting," "grateful," "stressful," "frustrating," "challenging," "instructive," "valuable," "difficult," "unpleasant," and "time-consuming." Of the 10 adjectives, students were allowed to choose a maximum of two (ie, the adjectives that best described their idea of treating patients with TMD and orofacial pain).

\section{Statistical Methods}

Mean values and SDs were calculated for all variables unless otherwise stated. A paired samples $t$ test analyzed differences over time for all items within each class. Between-class comparisons at the different time points were made with an independent samples $t$ test for all items. The variables were normalized and the relative changes were calculated with baseline being 100\%. Independent samples $t$ tests were applied to test for significant differences between classes in the mean values of the relative changes for all items. All $t$ test analyses were adjusted for multiple comparisons using Bonferroni correction to reduce the risk of type II errors. Pearson chi-square test was used to investigate if there were any significant associations between time (how long the student has been studying) and attitudes toward treating TMD patients. A $P$ value $<.05$ was considered to indicate statistical significance.

Binary logistic regression analyses were used to assess whether achieved competencies and satisfaction after the education could predict results of the course examination and the course evaluation. All statistical analyses were performed two-tailed at a significance level of $5 \%$. The Statistical Package for the Social Science for Windows, version 20 (SPSS, IBM) was used for all calculations.

\section{Results}

\section{First Hypothesis}

Anatomy and Physiology. In each class, the ratings of all items in achieved competencies and satisfaction increased significantly between the start of semester seven and the end of semester eight (paired samples $t$ test: $P<.001$; Table 2).

Clinical Training. In each class, the ratings of all items in achieved competencies and satisfaction increased significantly between the start of semester seven and the end of semester eight (paired samples $t$ test: $P<.001$; Table 2).

Attitudes. Table 3 presents attitudes toward treating patients with TMD and orofacial pain in each class. All adjectives apart from "unpleasant" were chosen by the students at some point. The two most commonly chosen adjectives in both classes were "challenging" and "interesting." A significant change in choice of the adjective "difficult" was observed in 
Table 2 Results of the Self-Evaluation in the Two Classes (2011/2012 and 2012/2013) of Achieved Competencies and Satisfaction at the Start of Semester Seven and After the Clinical Course at the End of Semester Eight

\begin{tabular}{|c|c|c|c|c|}
\hline & \multicolumn{2}{|c|}{ Class $2011 / 2012$} & \multicolumn{2}{|c|}{ Class $2012 / 2013$} \\
\hline & $\begin{array}{l}\text { Semester seven } \\
\quad(n=50)\end{array}$ & $\begin{array}{l}\text { Semester eight } \\
\quad(n=49)\end{array}$ & $\begin{array}{l}\text { Semester seven } \\
\quad(n=51)\end{array}$ & $\begin{array}{l}\text { Semester eight } \\
\quad(n=51)\end{array}$ \\
\hline Respondents & 50 & 43 & 49 & 40 \\
\hline \multicolumn{5}{|l|}{ Anatomy and physiology } \\
\hline \multicolumn{5}{|l|}{ TMJ } \\
\hline Achieved competence & $6.31 \pm 1.42$ & $8.66 \pm 1.29^{\star}$ & $6.06 \pm 1.49$ & $8.44 \pm 0.90^{\star}$ \\
\hline Satisfaction & $5.84 \pm 2.23$ & $8.67 \pm 1.44^{*}$ & $5.46 \pm 2.08$ & $8.71 \pm 1.23^{*}$ \\
\hline \multicolumn{5}{|l|}{ Jaw muscles } \\
\hline Achieved competence & $6.94 \pm 1.54$ & $8.64 \pm 1.33^{\star}$ & $6.24 \pm 1.53$ & $8.59 \pm 0.95^{\star}$ \\
\hline Satisfaction & $6.94 \pm 2.06$ & $8.42 \pm 1.83^{*}$ & $6.04 \pm 2.32$ & $8.93 \pm 1.15^{\star}$ \\
\hline \multicolumn{5}{|l|}{ Pain } \\
\hline Achieved competence & $5.24 \pm 1.77$ & $8.09 \pm 1.29^{\star}$ & $5.04 \pm 2.16$ & $8.03 \pm 1.20^{\star}$ \\
\hline Satisfaction & $4.54 \pm 2.05$ & $8.60 \pm 1.50^{*}$ & $4.79 \pm 2.32$ & $8.53 \pm 1.47^{\star}$ \\
\hline \multicolumn{5}{|l|}{ Clinical Training } \\
\hline \multicolumn{5}{|c|}{ Screening for TMD and orofacial pain } \\
\hline Achieved competence & $2.96 \pm 2.23$ & $8.80 \pm 1.29^{*}$ & $2.48 \pm 2.34$ & $8.66 \pm 1.11^{*}$ \\
\hline Satisfaction & $2.76 \pm 2.11$ & $8.66 \pm 2.00^{*}$ & $2.23 \pm 2.61$ & $9.00 \pm 1.10^{*}$ \\
\hline \multicolumn{5}{|l|}{ History and examination } \\
\hline Achieved competence & $4.24 \pm 2.05$ & $8.77 \pm 1.03^{*}$ & $3.27 \pm 2.40$ & $8.83 \pm 0.89^{\star}$ \\
\hline Satisfaction & $3.98 \pm 2.65$ & $9.07 \pm 1.24^{*}$ & $3.38 \pm 2.84$ & $9.22 \pm 0.99^{a}$ \\
\hline \multicolumn{5}{|l|}{ Diagnosis } \\
\hline Achieved competence & $3.42 \pm 2.03$ & $8.43 \pm 1.07^{\star}$ & $2.88 \pm 2.34$ & $8.83 \pm 0.97^{a}$ \\
\hline Satisfaction & $3.06 \pm 2.18$ & $8.67 \pm 1.51^{*}$ & $2.63 \pm 2.29$ & $9.05 \pm 1.12^{*}$ \\
\hline \multicolumn{5}{|l|}{ Prognosis } \\
\hline Achieved competence & $2.62 \pm 2.09$ & $8.39 \pm 1.26^{\star}$ & $2.32 \pm 2.33$ & $8.12 \pm 1.08^{*}$ \\
\hline Satisfaction & $2.32 \pm 2.07$ & $8.55 \pm 1.42^{*}$ & $2.51 \pm 2.49$ & $8.41 \pm 1.34^{*}$ \\
\hline \multicolumn{5}{|l|}{ Decision-making } \\
\hline Achieved competence & $2.86 \pm 2.07$ & $8.45 \pm 1.15^{\star}$ & $2.44 \pm 2.44$ & $8.41 \pm 1.00^{\star}$ \\
\hline Satisfaction & $2.43 \pm 1.90$ & $8.60 \pm 1.35^{\star}$ & $2.72 \pm 2.52$ & $8.43 \pm 1.20^{*}$ \\
\hline \multicolumn{5}{|c|}{ Evaluate treatment outcome } \\
\hline Achieved competence & $2.37 \pm 2.45$ & $8.36 \pm 1.31^{*}$ & $2.08 \pm 2.32$ & $8.24 \pm 1.20^{*}$ \\
\hline Satisfaction & $2.18 \pm 2.28$ & $8.58 \pm 1.44^{*}$ & $1.98 \pm 2.42$ & $8.37 \pm 1.32^{*}$ \\
\hline
\end{tabular}

*Significant increase compared with the start of semester seven in the same class $(P<.001)$. Each value expressed as mean \pm SD.

the first class (2011/2012), where the number of students choosing this adjective in the second evaluation (3) was significantly lower than in the first evaluation (11) (Pearson chi-square: $P<.05$ ). No other significant associations were observed in the first class (Pearson chi-square: $P>.05$; data not presented).

In the second class (2012/2013), no significant changes in attitudes were observed between the beginning of semester seven and the end of semester eight (Pearson chi-square: $P>.05$ ).

\section{Second Hypothesis}

Anatomy and Physiology. No significant differences in competencies and satisfaction were observed between the classes at the beginning of semester seven or at the end of semester eight (independent samples $t$ test: $P>.05$, Table 2).
No significant differences in relative increases in competencies and satisfaction were observed between classes (independent samples $t$ test: $P>.05$ ).

Clinical Training. At the beginning of semester seven and at the end of semester eight, there were no significant differences in competencies and satisfaction between the classes (independent samples $t$ test: $P>.05$; Table 2).

No significant differences in relative increases in competencies and satisfaction were observed between the classes for any items (independent samples $t$ test: $P>$.05).

Attitudes. A comparison was made between classes at the beginning of semester seven and also after the preclinical course, and there were no significant differences between classes. (Pearson chi-square: $P>.05$; Table 3). 


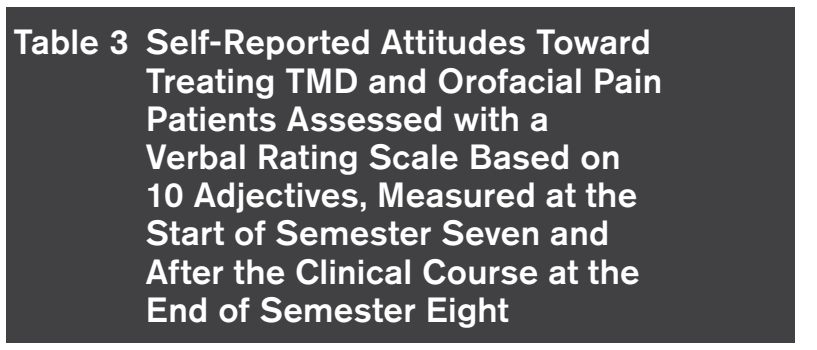

\begin{tabular}{cccccc}
\cline { 5 - 6 } $\begin{array}{c}\text { Semester } \\
\text { seven }\end{array}$ & $\begin{array}{c}\text { Semester } \\
\text { eight }\end{array}$ & & $\begin{array}{c}\text { Semester } \\
\text { seven }\end{array}$ & $\begin{array}{c}\text { Semester } \\
\text { eight }\end{array}$ \\
\hline
\end{tabular}

\begin{tabular}{lcccc}
\hline Interesting & & & & \\
Yes & 21 & 13 & 22 & 13 \\
No & 29 & 31 & 25 & 28 \\
Grateful & & & & \\
Yes & 6 & 8 & 6 & 8 \\
No & 44 & 36 & 41 & 33
\end{tabular}

Stressful

$\begin{array}{lllll}\text { Yes } & 1 & 1 & 0 & 1\end{array}$

$\begin{array}{lllll}\text { No } & 49 & 43 & 47 & 40\end{array}$

Frustrating

$\begin{array}{lcccc}\text { Yes } & 3 & 3 & 3 & 1 \\ \text { No } & 47 & 41 & 44\end{array}$

Challenging

$\begin{array}{lllll}\text { Yes } & 26 & 22 & 24 & 18\end{array}$

No 24

Instructive

Yes 14

No 36

Valuable

No

Difficult

$\begin{array}{llllc}\text { Yes } & 11 & 3^{*} & 11 & 6 \\ \text { No } & 39 & 41 & 36 & 35\end{array}$

Unpleasant

Yes

No

$-$

Time-

consuming

Yes

No

46

$14 \quad 15$

15
29

15

32

15

18

19

28

23

*Significant decrease compared with the start of semester seven in the same class $(P<.05)$.

\section{Third Hypothesis}

A total of 47 out of 50 students in the first class (2011/2012) and 49 of 50 students in the second class (2012/2013) passed the course examination. Performing a binary logistic regression analysis to predict the outcome of the course examination based on the achieved competencies and satisfaction with the education was not possible.

\section{Additional Outcomes}

Table 4 presents the results of the students' self-evaluation of how well the course goals were fulfilled.

\section{Discussion}

The main findings of this study are that (1) students in both classes considered themselves highly competent; (2) similar levels of achieved competencies were reached independent of whether the RDC/TMD or DC/TMD was used; and (3) no predictors for the outcome of the examination could be identified due to the high number of students that passed, which made a binary logistic analysis not possible to perform.

\section{Testing of the First Hypothesis}

A significant increase in achieved competencies and satisfaction with the education in TMD and orofacial pain from the start to the end of the training was observed for all self-evaluation items in both classes, which corroborates the first hypothesis. This also confirms that the Malmö model and the curriculum of the recently expanded preclinical and clinical courses in TMD and orofacial pain improve skills. ${ }^{3}$

\section{Testing of the Second Hypothesis}

At the start of training (semester seven), there were no significant differences in competencies and satisfaction between classes; however, some differences would have been expected due to varying levels of precourse competencies. Importantly, no significant differences were observed between classes in competencies and satisfaction at the end of the clinical course in semester eight. This implies that the recent expansion in pain education allows all students to reach the same level of competence, independent of prior experience. It also confirms the second hypothesis (that no differences would occur between classes) and implies that an evaluation of the education in TMD and orofacial pain in precourse semesters could be beneficial.

It is impossible to give all students the exact same information despite the same core curriculum. Teachers have different experiences and education. Students have varied theoretic knowledge and are exposed to different scenarios in the clinical environment. This could explain variations in learning opportunities, the choice of topics that are discussed during clinical hours, and the depth of the discussions. To avoid excessive fluctuations, teacher meetings are held during the semesters to calibrate each teacher. This seems to be a workable, although not flawless, method for providing all students access to the same information. Nevertheless, from one year to another, small changes are always made in ongoing efforts to improve education. Decisions to make changes in the education protocol are based on examination results, course evaluations, and teachers' practical experience. But given that the core curriculum is standardized, small fluctuations within or between groups seem to be of less importance. 


\begin{tabular}{|c|c|c|c|c|c|c|}
\hline & \multicolumn{2}{|c|}{2011} & \multicolumn{2}{|c|}{2012} & \multicolumn{2}{|c|}{2013} \\
\hline & $\begin{array}{c}\text { NRS } \\
1-3\end{array}$ & $\begin{array}{l}\text { NRS } \\
4-5\end{array}$ & $\begin{array}{c}\text { NRS } \\
1-3\end{array}$ & $\begin{array}{l}\text { NRS } \\
4-5\end{array}$ & $\begin{array}{c}\text { NRS } \\
1-3\end{array}$ & $\begin{array}{l}\text { NRS } \\
4-5\end{array}$ \\
\hline \multicolumn{7}{|l|}{ Semester 7} \\
\hline Explain TMD and headache, as well as its incidence, onset, course, and treatment & 0 & 91.6 & 2.5 & 95 & - & - \\
\hline $\begin{array}{l}\text { Explain the pharmacologic mechanisms of peripherally and centrally acting } \\
\text { analgesics (including adverse effects) and its efficacy in the treatment of TMD }\end{array}$ & 25 & 66.7 & 12.5 & 82.5 & - & - \\
\hline $\begin{array}{l}\text { Assess and inform about treatment possibilities and restrictions from both a } \\
\text { professional and subjective perspective on oral prosthetic rehabilitation and } \\
\text { TMD treatment, as well as plan and execute these }\end{array}$ & 8.3 & 87.5 & 5 & 95 & - & - \\
\hline $\begin{array}{l}\text { Apply knowledge of the function of the stomatognathic system in conjunction with } \\
\text { oral prosthetic rehabilitation and, during treatment, be able to diagnose, } \\
\text { make a prognosis, and treat TMD }\end{array}$ & 8.3 & 87.7 & 5 & 95 & - & - \\
\hline Prescribe peripherally and centrally acting analgesics for oral pain conditions & 45.8 & 50 & 25 & 75 & - & - \\
\hline \multicolumn{7}{|l|}{ Semester 8} \\
\hline $\begin{array}{l}\text { Analyze TMD and its prevalence, onset, course, diagnosis, prognosis, and } \\
\text { treatment in children and adolescents }\end{array}$ & - & - & 8.3 & 87.5 & 2.6 & 94.7 \\
\hline Routinely identify TMD patients in clinical practice & - & - & 12.5 & 87.5 & 5.3 & 84.2 \\
\hline
\end{tabular}

"Evaluation with anchor definitions "not fulfilled" and "fulfilled."

\section{Testing of the Third Hypothesis}

Since the vast majority of students passed the examination, a binary logistic regression analysis was not possible to perform. Therefore, it was not possible to identify any predictors for the outcome of the examination. Even so, the data are consistent with the results of the course examinations. The students appear to have the ability to evaluate themselves in a way that reflects their true capacity. Throughout their dental education, the students continuously evaluate themselves and the tasks that they have performed, and they receive feedback on their evaluations from teachers. In this way, students become familiar with their strengths and weaknesses and are thus well prepared to analyze their management of various clinical situations. Overall, undergraduate dental students are well educated and consider themselves prepared to identify, treat, and assess treatment of patients with TMD and orofacial pain.

\section{Clinical Relevance}

All clinical cases in the theoretic education are supposed to be clinically relevant and reflect a possible TMD or orofacial pain patient. To accurately assess the case, students must have knowledge of anatomy, physiology, pain mechanisms, pharmacology, and treatment options. To be able to answer patients' questions and give reassuring information, the dentist must have the requisite knowledge and understanding.

The Miller triangle describes the various levels of competence and performance..$^{10}$ The triangle is divided into blocks and each building block is the foundation for the next level. "Knows," "knows how," and "shows how"-in ascending order-represent levels of competence. The topmost stage, "does," concerns performance and requires analytic skills to determine how to apply competencies in everyday situations. It also requires an ability to reflect over how the competence was applied as a part of the learning experience.

When students reach the performance level, they are more likely to be able to apply their competencies in a wider range of situations-that is, if they meet a patient with TMD or other type of orofacial pain in general practice that reminds them of the studied cases, they will be able to combine their previous knowledge and competencies with new knowledge and competencies that they have independently sought and analyzed in order to devise an individualized treatment plan. In other words, it is important that the students remember and have confidence in how to manage TMD and orofacial pain patients when they are educated dentists and that they maintain this capacity throughout the years. Students are therefore encouraged to stay up to date, be source critical, and choose treatment options that are reliable and valid. As an example, the Swedish National Guidelines are central when discussing treatment options with students. ${ }^{11}$

This study has focused on students' experiences of their achieved competencies during their TMD and orofacial pain education. It would also be interesting to know how they would assess their competencies a couple of years into their profession. The Vallon and Nilner study addressed this, and they concluded that postgraduates with 5 to 6 years of experience as general dental practitioners still had a positive attitude and high satisfaction regarding their undergraduate education in TMD and orofacial pain. ${ }^{5}$ 
In some cases, self-evaluations may report decreased competence over time. The discovery that some things are more complex than previously thought could explain a decrease in self-perceived ability.

\section{Limitations}

It is difficult to determine the amount of time spent solely on pain education since the PBL approach involves learning in several different dental fields at the same time, in both theory and practice. Therefore, the number of hours of pain education in undergraduate dental education at Malmö University cannot be compared with those reported by Watt-Watson et al. ${ }^{1}$

There were a few dropouts in the present study, but due to the low number in each class it most likely did not bias the results. Another indicator for this is the fact that the results were still significant after the adjustment for multiple comparisons.

Another study limitation was how and when the DC/TMD was introduced to the students. Some ambiguities were revealed when testing the DC/TMD in the student clinic for the first time, which can be expected with something new. For example, the algorithm for diagnoses was introduced a little later in the study since it had not been finished when semester seven began. However, no significant difference was observed between the two classes regarding students' competencies and satisfaction in their ability to take a history and perform an examination or to diagnose a patient.

It would also be interesting to know how postgraduates who have been taught the DC/TMD compare with postgraduates taught the RDC/TMD in their attitude toward and competence in TMD and orofacial pain a couple of years after graduation. This is an interesting question, since one of the aims of the DC/TMD was to make it easier for the general practitioner to examine and diagnose TMD, and will be investigated in a future follow-up study.

\section{Conclusions}

Students' achieved competencies and satisfaction with pain education increased over the seventh and eighth semesters in a spiral fashion similar to the Malmö model and the design of the undergraduate education curriculum. No differences in competencies and satisfaction were observed between the two classes after the clinical course in semester eight.
The recently expanded pain education at Malmö University has allowed all students to reach the same level of competence, independent of previous competencies. This developed method of interactive pain education, used in line with the Malmö model, appears to implement pain education successfully in undergraduate dental education. ${ }^{3}$ The results from this study emphasize the importance of continuously evaluating and improving pain education in undergraduate dental education.

\section{Acknowledgments}

The authors declare no conflicts of interest.

\section{References}

1. Watt-Watson J, McGillion M, Hunter J, et al. A survey of prelicensure pain curricula in health science faculties in Canadian universities. Pain Res Manag 2009;14:439-444.

2. Sessle B. Unrelieved pain: A crisis. Pain Res Manag 2011;16:416-420.

3. Rohlin M, Petersson K, Svensäter G. The Malmö model: A problem-based learning curriculum in undergraduate dental education. Eur J Dent Educ 1998;2:103-114.

4. LeResche L. Epidemiology of temporomandibular disorders: Implications for the investigation of etiologic factors. Crit Rev Oral Biol Med 1997;8:291-305.

5. Vallon D, Nilner M. Undergraduates' and graduates' perception of achieved competencies in temporomandibular disorders and orofacial pain in a problem-based dental curriculum in Sweden. Eur J Dent Educ 2009;13:240-247.

6. Alsafi Z, Michelotti A, Ohrbach R, Nilner M, List T. Achieved competencies in temporomandibular disorders/orofacial pain: A comparison between two dental schools in Europe. Eur J Dent Educ 2015;19:161-168.

7. Nilsson IM. Reliability, validity, incidence and impact of temporomandibular pain disorders in adolescents. Swed Dent J Suppl 2007;(183):7-86.

8. Dworkin SF, LeResche L. Research diagnostic criteria for temporomandibular disorders: Review, criteria, examinations and specifications, critique. J Craniomandib Disord 1992;6:301-355.

9. Schiffman E, Ohrbach R, Truelove E, et al. Diagnostic Criteria for Temporomandibular Disorders (DC/TMD) for clinical and research applications: Recommendations of the International RDC/TMD Consortium Network and Orofacial Pain Special Interest Group. J Oral Facial Pain Headache 2014;28:6-27.

10. Miller GE. The assessment of clinical skills/competence/performance. Acad Med 1990;65:S63-S67.

11. National Guidelines for Adult Dental Care 2011: The National Board of Health and Welfare [in Swedish]. Stockholm: Socialstyrelsen, 2011. 